Please do not remove this page

RMIT

UNIVERSITY

\title{
A gallium-based magnetocaloric liquid metal ferrofluid
}

Alves De Castro, Isabela; Chrimes, Adam; Zavabeti, Seyed Mohammad Ali; Berean, Kyle; Carey, Benjamin; Zhuang, Jincheng; Du, Yi

https://researchrepository.rmit.edu.au/esploro/outputs/9921863169601341/filesAndLinks?institution=61RMIT_INST\&index=null

Alves De Castro, I., Chrimes, A., Zavabeti, S. M. A., Berean, K., Carey, B., Zhuang, J., Du, Y., Dou, S., Suzuki, K., Shanks, R., Nixon-Luke, R., Bryant, G., Khoshmanesh, K., Kalantar Zadeh, K., \& Daeneke, T. (2017). A gallium-based magnetocaloric liquid metal ferrofluid. Nano Letters, 17(12), 7831-7838.

https://doi.org/10.1021/acs.nanolett.7b04050

Document Version: Accepted Manuscript

Published Version: https://doi.org/10.1021/acs.nanolett.7b04050

Repository homepage: https://researchrepository.rmit.edu.au

(c) 2017 American Chemical Society.

Downloaded On 2023/04/26 21:50:14 +1000 
Thank you for downloading this document from the RMIT Research Repository.

The RMIT Research Repository is an open access database showcasing the research outputs of RMIT University researchers.

RMIT Research Repository: http://researchbank.rmit.edu.au/

\section{Citation:}

Alves De Castro, I, Chrimes, A, Zavabeti, S, Berean, K, Carey, B, Zhuang, J, Du, Y, Dou, S, Suzuki, K, Shanks, R, Nixon-Luke, R, Bryant, G, Khoshmanesh, K, Kalantar Zadeh, $\mathrm{K}$ and Daeneke, T 2017, 'A gallium-based magnetocaloric liquid metal ferrofluid', Nano Letters, vol. 17, no. 12, pp. 7831-7838.

See this record in the RMIT Research Repository at:

https://researchbank.rmit.edu.au/view/rmit:47068

Version: Accepted Manuscript

Copyright Statement:

(C) 2017 American Chemical Society.

Link to Published Version:

https://dx.doi.org/10.1021/acs.nanolett.7b04050 
Please cite as:

de Castro, I.A., Chrimes, A.F., Zavabeti, A., Berean, K.J., Carey, B.J. Zhuang, J., Du, Y., Dou, S.X., Suzuki, K., Shanks, R.A., Nixon-Luke, R., Bryant, G., Khoshmanesh, K., Kalantar-zadeh, K., Daeneke, T. “A Gallium-Based Magnetocaloric Liquid Metal Ferrofluid" NanoLetters Nano Lett., 17, 7831-7838 (2017). DOI: 10.1021/acs.nanolett.7b04050 


\section{A Gallium-based Magnetocaloric Liquid Metal}

\section{Ferrofluid}

Isabela A. de Castro, ${ }^{1}$ Adam F. Chrimes, ${ }^{1}$ Ali Zavabeti, ${ }^{1}$ Kyle J. Berean, ${ }^{1}$ Benjamin J. Carey, ${ }^{1}$ Jincheng Zhuang, ${ }^{2}$ Yi Du, ${ }^{2}$ Shi X. Dou, ${ }^{2}$ Kiyonori Suzuki, ${ }^{3}$ Robert A. Shanks, ${ }^{4}$ Reece Nixon-Luke, ${ }^{5}$ Gary Bryant, ${ }^{5}$ Khashayar Khoshmanesh, ${ }^{1}$ Kourosh Kalantar-zadeh, ${ }^{1 *}$ and Torben Daeneke ${ }^{l^{*}}$

1. School of Engineering, RMIT University, Melbourne, VIC 3001, Australia

2. Institute for Superconducting and Electronic Materials, University of Wollongong, Wollongong, NSW 2500, Australia

3. Department of Materials Science and Engineering, Monash University, Clayton, VIC 3168, Australia

4. School of Science, RMIT University, Melbourne, VIC 3001, Australia

5. Centre for Molecular and Nanoscale Physics, School of Science, RMIT University, Melbourne, VIC 3001, Australia 
ABSTRACT: We demonstrate a magnetocaloric ferrofluid based on a gadolinium saturated liquid metal matrix, using a gallium based liquid metal alloy as the solvent and suspension medium. The material is liquid at room temperature, while exhibiting spontaneous magnetization and a large magnetocaloric effect. The magnetic properties were attributed to the formation of gadolinium nanoparticles suspended within the liquid gallium alloy, which acts as a reaction solvent during the synthesis. High nanoparticle weight fractions exceeding $2 \%$ could be suspended within the liquid metal matrix. The liquid metal ferrofluid shows promise for magnetocaloric cooling due to its high thermal conductivity and its liquid nature. Magnetic and thermoanalytic characterizations reveal that the developed material remains liquid within the temperature window required for domestic refrigeration purposes, which enables future fluidic magnetocaloric devices. Additionally, the observed formation of nanometer sized metallic particles within the supersaturated liquid metal solution has general implications for chemical synthesis and provides a new synthetic pathway towards metallic nanoparticles based on highly reactive rare earth metals.

KEYWORDS: magnetic cooling, galinstan, metallic ferrofluid, metallic nanoparticles, gadolinium, liquid metal reaction environment

The interest in magnetocaloric materials with a Curie point near room temperature has increased significantly due to their potential use in heat pumps and refrigeration devices. ${ }^{1-3}$ The magnetocaloric effect (MCE) occurs in materials that can undergo adiabatic demagnetisation when exposed to a decreasing magnetic field. ${ }^{4}$ During this process, phonons couple to the magnetic domains, leading to demagnetisation and cooling. ${ }^{5}$ This effect has been exploited in 
cooling applications and is widely applied in the field of low temperature physics. ${ }^{1}$ Prototypes of domestic magnetocaloric refrigerators have been developed, with the ultimate aim to create efficient refrigeration technologies that do not rely on working fluids such as fluorocarbons (CFCs) or hydrocarbons that may harm the environment. ${ }^{4,6}$

In most magnetocaloric based devices the caloric material is utilized in the form of a porous solid structure which is in contact with a working-fluid that facilitates heat exchange., 7 Recently, significant work has been dedicated to develop magnetocaloric ferrofluids, which consist of magnetocaloric nanoparticles suspended in a suitable solvent. ${ }^{8}$ The advantage of utilizing ferrofluids is the improved thermal contact between the working fluid and the magnetocaloric material. ${ }^{8}$ The ideal material for a MCE should exhibit a high magnetic entropy change $\Delta \mathrm{S}$, defined as the change in entropy of a material under isothermal conditions when the external field is changed, and a large adiabatic temperature difference $\left(\Delta \mathrm{T}_{\mathrm{ad}}\right)$ for heat exchange. ${ }^{1}$ The working fluid should feature suitable melting and boiling points, a high thermal conductivity and low viscosity to ensure a fast temperature response and efficient heat transfer. ${ }^{9}$

Room temperature liquid metals such as gallium and its alloys (eutectic gallium-indium (EGaIn) and eutectic gallium-indium-tin (galinstan)) are predicted to be a superior alternative working fluid for magnetocaloric devices, owing to their superior thermal conductivity, relatively high heat capacity and low viscosity.$^{10}$ These properties are expected to lead to more efficient heat exchange when compared with regular fluids used for magnetocaloric devices which are typically based on water and ethylene glycol..$^{10}$ These alloys feature low toxicity and extremely high boiling points $\left(>1300^{\circ} \mathrm{C}\right)$, making them ideal candidates for applications in high temperature environments, and for cooling applications that require operation over a wide range of working temperatures, which might be inaccessible using traditional solvents. ${ }^{10-12}$ The high 
surface tension of liquid galinstan (7-10 times higher than water) suggests that the liquid may accompany significantly higher mass fractions of suspended nanoparticles, which may enable the design of improved magnetocaloric ferrofluids. ${ }^{8,10}$ The inclusion of suspended nanoparticles with strong MCE is a necessity for designing magnetocaloric ferrofluids based on gallium alloys. It is known that gadolinium (Gd) and some of its alloys feature strong MCE near room temperature. ${ }^{4}$ ${ }^{6,13-15} \mathrm{Gd}$ solubility has been reported to be below $0.2 \mathrm{wt} \%$ in liquid $\mathrm{Ga}$ at ambient temperatures. ${ }^{16}$ However, the nature and distribution of the alloyed Gd inside the liquid metal has yet to be identified.

Herein we investigate liquid metal composites based on the eutectic mixture of gallium, indium and tin which is better known by the trade name Galinstan ${ }^{\circledR}$ (Ga:In:Sn 68:22:10 wt\%) with incorporated Gd. We recently introduced galinstan as a reaction solvent for the synthesis of ultrathin metal oxide nanosheets which can be isolated from the liquid metal air interface. ${ }^{17,}{ }^{18}$ In our previous work, we developed methods that allow the preparation of galinstan based alloys containing approximately $1 \mathrm{wt} \%$ of added transition, post-transition and rare earth metals. For this study, we develop quaternary mixtures containing galinstan and various weight fractions of Gd $(0.2$ to $2.3 \mathrm{wt} \%)$. The synthesis of these materials is effectively achieved utilizing manual grinding of micron sized Gd particles within the liquid melt in an inert atmosphere. The investigated concentration range is around and above the expected solubility of Gd within the Ga based liquid melt. ${ }^{16}$ Assuming a similar solubility of Gd in galinstan, excess Gd is expected to precipitate within the liquid melt, forming a colloid, effectively creating a liquid metal ferrofluid (see Figure 1a). In this process the liquid metal effectively assumes the role of a reaction solvent. The presence of elemental gadolinium nanoparticles is expected to be advantageous due 
to the increased interfacial surface area between the magnetocaloric material and the carrying fluid, which is expected to facilitate more efficient heat exchange. ${ }^{8,19}$

A schematic representation of the magnetocaloric cooling process is presented in Figure $\mathbf{1 b}$. The Gd:galinstan melt at room temperature (RT) (step I) is exposed to a magnetic field, leading to a temperature increase during magnetization (step II). The energy is released to the environment in the form of heat. Once the magnetized metallic ferrofluid has cooled down sufficiently it may be moved out of the magnetic field (step III). Demagnetization of the metallic ferrofluid is then accompanied by a temperature drop, enabling magnetic cooling.

(a)

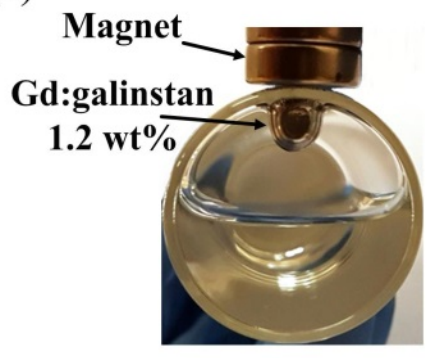

(b)

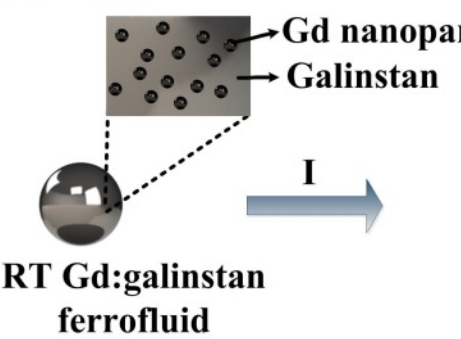

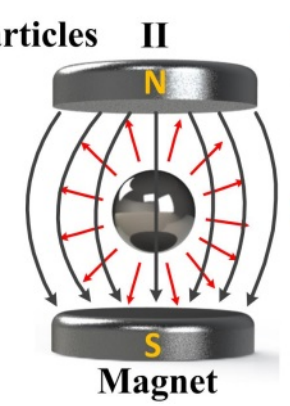

$\longrightarrow$ Heat flow Magnetic field

III

Cold Gd:galinstan ferrofluid

Figure 1 - (a) A droplet of the $1.2 \mathrm{wt} \% \mathrm{Gd}$ :galinstan capable to support its own mass against gravity in the presence of a permanent magnet. The metal is kept in a solution of $\mathrm{HCl} 0.01$ mol L ${ }^{-1}$ to avoid the formation of a surface oxide layer. ${ }^{20}$ (b) Schematic of the magnetocaloric refrigeration process based on our developed magnetic liquid metal. Step I shows the Gd:galinstan ferrofluid at RT. Step II shows the increase of the metals temperature caused by the exposure of the Gd nanoparticles to a magnetic field. The energy is consequently released to the environment as heat. Step III shows the ferrofluid when it leaves the magnetic field, featuring a temperature lower than that in step I. 
Manual grinding assisted alloying of gadolinium into galinstan was achieved at room temperature and under inert atmosphere, utilizing mortar and pestle. Micrometer sized Gd particles $(\mathrm{d} \sim 425 \mu \mathrm{m})$ were successfully dissolved in galinstan, leading to a liquid metal with homogeneous appearance. Composites containing between 0.2 and $2.3 \mathrm{wt} \% \mathrm{Gd}$ were synthesized, with all products retaining their fluidic properties. The developed process is facile and scalable, and no additional chemicals or processes were found to be necessary for the successful loading of $\mathrm{Gd}$ into the galinstan matrix.

Figure 1a shows an image of a Gd:galinstan sample containing $1.2 \mathrm{wt} \%$ of $\mathrm{Gd}$. The composites evidently possess strong macroscopic magnetic properties, being able to support its own mass against gravity in the presence of a magnetic field. The observation of macroscopic magnetic properties suggests the presence of solid domains within the liquid melt, since magnetic ordering under ambient conditions usually occurs in the solid aggregate state. ${ }^{21}$ The implied presence of solid Gd particles is in good agreement with the reported low solubility of gadolinium in gallium. ${ }^{16}$ The sample containing $0.2 \mathrm{wt} \%$ of $\mathrm{Gd}$ possessed no macroscopic magnetic properties, indicating that for this composite most of the Gd has been truly dissolved into the metal.

Differential scanning calorimetry (DSC) revealed that pure galinstan as well as two samples containing 0.2 and $1.2 \mathrm{wt} \%$ Gd feature similar solidus temperatures, indicating that the liquid phase behaves predominantly like pure galinstan (Figure 2). The solidus temperature is characterized by a broad endothermic peak located at $13.2^{\circ} \mathrm{C}$ for pure galinstan and $13.2^{\circ} \mathrm{C}$ and $13.7^{\circ} \mathrm{C}$ for galinstan containing 0.2 and $1.2 \mathrm{wt} \% \mathrm{Gd}$, respectively. The liquidus temperatures feature larger variations for the three investigated melts. The cooling scan of pure galinstan reveals that the crystallization process occurs in two distinct nucleation processes starting at 
$3.3^{\circ} \mathrm{C}$. This pre-solidification process is followed by a sharp peak at $-14.2^{\circ} \mathrm{C}$ associated with the complete crystallization of galinstan. The presence of a pre-solidification process followed by the crystallization of galinstan at lower temperatures is in agreement with a previous report by Jin et $a l .{ }^{22}$ For the sample containing $0.2 \mathrm{wt} \%$ of $\mathrm{Gd}$, crystallization occurs at $-17.9^{\circ} \mathrm{C}$. With an increase of the $\mathrm{Gd}$ content to $1.2 \mathrm{wt} \%$, a sharp crystallization peak is observed at $-21.9^{\circ} \mathrm{C}$. Neither of the Gd containing metallic melts showed a pre-solidification peak. Overall the thermoanalytic characterization revealed that the liquidus temperature of our synthesized galinstan based liquid decreased with the addition of $\mathrm{Gd}$, which significantly suppressed crystallization, leading to solidification of the mixture at temperatures around $-18^{\circ} \mathrm{C}$ and $-22^{\circ} \mathrm{C}$ for the materials containing 0.2 and $1.2 \% \mathrm{Gd}$, respectively. Furthermore these results reveal that the developed metallic materials remain liquid within the typical operating temperatures relevant to domestic refrigeration applications. The results highlight that the addition of small quantities of gadolinium allow the suppression of the crystallization temperature of galinstan, widening the temperature window for practical fluidic applications which may be relevant to applications beyond magnetocalorics.

Scanning electron microscopy (SEM) based imaging and energy-dispersive X-ray spectroscopy (EDXS) elemental analysis were conducted in order to characterize the distribution of the added Gd and identify expected solid inclusions within the melts Figure SI 1. Despite the inferred presence of solid gadolinium containing crystals, based on the observed macroscopic magnetic properties, the elemental distribution within the liquid metal was found to be uniform, indicating that no larger $\mathrm{Gd}$ rich domains are present within the resolution of the measurement $(\sim 10 \mathrm{~s}$ of $\mathrm{nm})$. This indicates that the larger $\mathrm{Gd}$ precursor particles $(\sim 425 \mu \mathrm{m})$ were fully dissolved within the liquid metal during the grinding stage. 


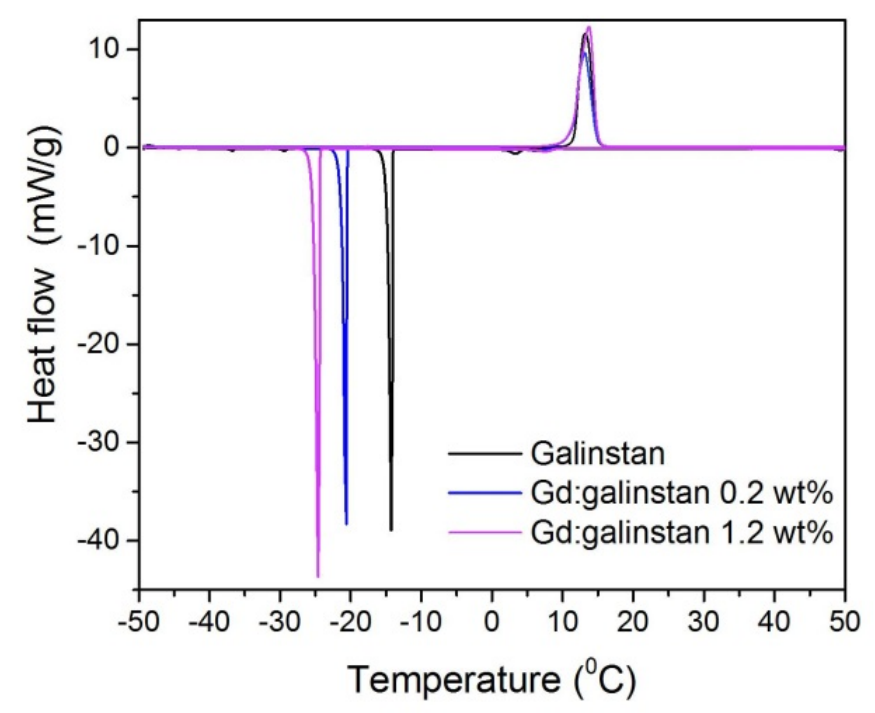

Figure 2. DSC curves for the 0.2 and $1.2 \mathrm{wt} \% \mathrm{Gd}$ :galinstan metallic suspensions and pure galinstan for temperatures between 50 and $-50^{\circ} \mathrm{C}$.

Small-angle X-ray scattering (SAXS) was performed to confirm the presence and determine the size distribution of suspended Gd particles within the liquid melt. Comparison of the pure galinstan sample with the sample containing $1.2 \mathrm{wt} \% \mathrm{Gd}$ revealed the presence of small nanoparticles within the Gd containing liquid metal, as indicated in the Guinier plot (Figure 3a). The radius of gyration $(\mathrm{Rg})$ was calculated using Guinier analysis ( $\mathrm{Rg}=48 \AA$ ). The radius of gyration is directly related to the radius of a spherical particle $\left(\mathrm{Rg}^{2}=3 / 5 \mathrm{R}^{2}\right)$, which leads to an estimated diameter of $\sim 12.6 \mathrm{~nm}$ for the Gd particles, assuming that they are spherical. ${ }^{23}$

The presence of Gd nanoparticles within the melt was further confirmed using High resolution transmission electron microscopy (HRTEM) analysis. Ultracentrifugation at 50,000 rcf was utilized to separate the solid particles from the liquid galinstan matrix. The density of gadolinium $\left(7.90 \mathrm{~g} \mathrm{~cm}^{-3}\right)^{24}$ is higher than that of galinstan $\left(6.36 \mathrm{~g} \mathrm{~cm}^{-3}\right),{ }^{12}$ allowing efficient separation. 
Centrifugation of a small droplet of the metallic suspension was conducted within a larger volume of dimethylformamide (DMF). The separated Gd nanoparticles were found to enter the organic phase, which could then be decanted and analyzed. HRTEM imaging confirmed the presence of spherical particles with diameters in the order of 7 to $17 \mathrm{~nm}(12.7 \pm 3.5 \mathrm{~nm}$, see Figure SI-2), confirming the SAXS results. The isolated particles were found to have oxidized, featuring oxide shells with d spacings corresponding well to the (222), (440) and (622) planes of cubic $\mathrm{Gd}_{2} \mathrm{O}_{3}$ Figure 3c. ${ }^{25}$ Gadolinium metal is well known to oxidize rapidly in ambient atmosphere, leading to the formation of the $\mathrm{Gd}_{2} \mathrm{O}_{3}$ shell during sample preparation and the transfer of the TEM grid into the instrument. ${ }^{24}$ The gadolinium particles do not feature an oxide shell within the liquid melt due to the overall reducing conditions within the liquid metal. Overall, HRTEM analysis confirms that the nanoparticles present within the liquid melt are likely composed of elemental gadolinium, lending the liquid metal the observed macroscopic magnetic properties.

The presence of nanometer sized Gd particles itself is noteworthy and significant, since the micrometer sized precursor particles are unlikely to have been broken down to this size by simple manual grinding, due to the inherent limitations of mechanical grinding approaches that prohibit achieving nanoparticles with average dimensions below $100 \mathrm{~nm} \cdot{ }^{26,27}$ Instead, Gd has likely been dissolved within the liquid melt and precipitated to form small nanoparticles $(\sim 13$ $\mathrm{nm})$. While the solubility of Gd in galinstan is unknown, its solubility in liquid gallium has been investigated by Palenzona et al. and was found to be below $0.2 \mathrm{wt} \%$ at $29^{\circ} \mathrm{C}$, where it forms a gallium rich eutectic. ${ }^{16}$ Intermetallic compounds were not found to form at low $\mathrm{Gd}$ concentrations and ambient temperatures and are thus not expected to be present in our sample. ${ }^{16}$ In combination, our observations clearly highlight that ambient temperature liquid metals are 
suitable reaction environments for the synthesis of metallic nanomaterials, and that the precise mechanisms behind the formation of Gd nanoparticles should be studied in further detail. For the 1.2 and $2.3 \mathrm{wt} \% \mathrm{Gd}$ :galinstan sample the suspended $\mathrm{Gd}$ concentration reaches 0.5 and $0.9 \mathrm{~mol} \mathrm{~L}^{-1}$, respectively. Similar nanoparticle loading in aqueous suspensions can only be achieved when elaborate surface modification and stabilization strategies are employed. ${ }^{28}$ The finding highlights that low temperature liquid metals should be considered for nanomaterial synthesis alongside conventional molecular and ionic solvents.

(a)

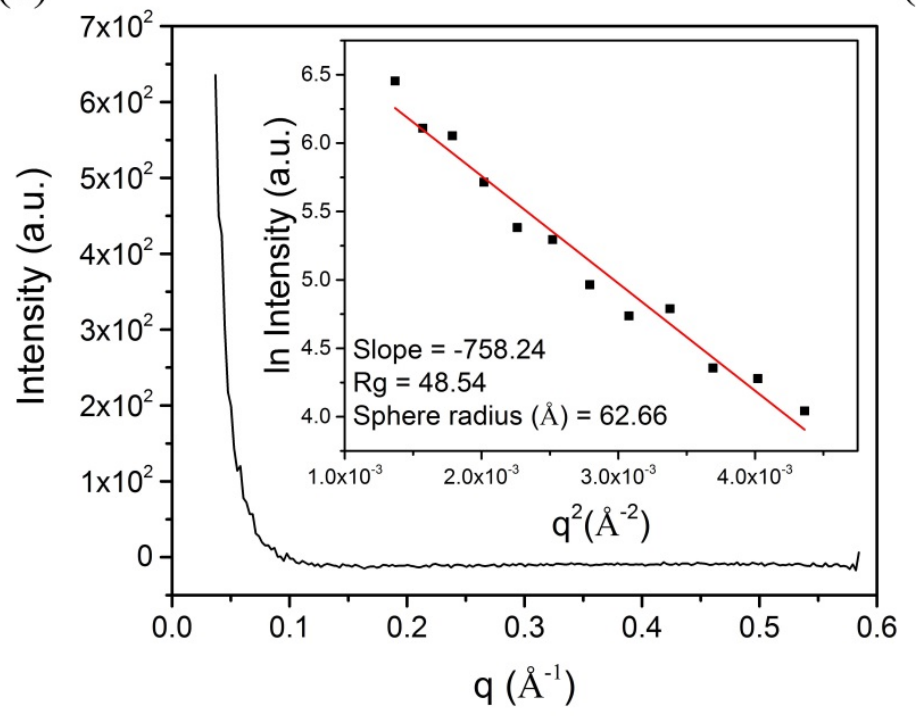

(b)

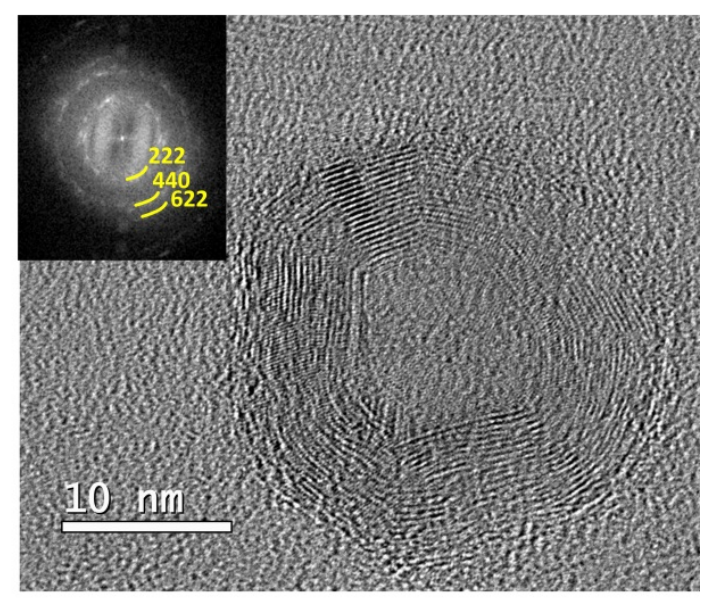

Figure 3. (a) SAXS spectrum of the $1.2 \mathrm{wt} \% \mathrm{Gd}$ :galinstan sample, the inset features the corresponding Guinier plot. (b) HRTEM of Gd nanoparticle obtained after ultracentrifugation of $1.2 \mathrm{wt} \% \mathrm{Gd}$ :galinstan showing the oxide shell with lattice fringes corresponding to cubic $\operatorname{Gd}_{2} \mathrm{O}_{3}$. The inset features fast Fourier transform (FFT) of the HRTEM image with indicated lattice planes. 
Magnetic hysteresis loops (MHLs) were measured at temperatures ranging from $300 \mathrm{~K}$ to $5 \mathrm{~K}$, using magnetic field strengths up to $2 \mathrm{~T}$, with a ramping rate of $100 \mathrm{Oe} \mathrm{s}^{-1}$. Both pure Gd powder and the metallic ferrofluid containing $1.2 \mathrm{wt} \%$ Gd in galinstan were investigated (Figure 4a and d). The MHL curves were normalized to the Gd mass in each sample to facilitate a direct comparison between the two materials. Both samples display similar magnetic properties, indicating that the magnetic properties of the liquid metal arise from elemental Gd nanoparticles. Soft ferromagnetic properties are indicated based on the completely reversible behavior at different temperatures, promising completely reversible magnetocaloric properties for the developed metallic suspensions.

No hysteresis was observed at $300 \mathrm{~K}$, Figure $\mathbf{4 b}$ and $\mathbf{e}$, however, a clear hysteresis loop is observed at cryogenic temperatures (i.e. $5 \mathrm{~K}, \mathrm{Hc}=118.5 \mathrm{Oe}$ ), Figure $\mathbf{4 c}$ and $\mathbf{f}$ for both pure $\mathrm{Gd}$ and the $1.2 \mathrm{wt} \%$ Gd:galinstan ferrofluid with the same coercivity value. The absence of ferromagnetic ordering at $300 \mathrm{~K}$ is in excellent agreement with the Curie temperature of Gd (293 K). ${ }^{15}$ The observed similarity between the two investigated samples, together with the HRTEM and SAXS results, strongly suggests that the observed magnetic properties of the synthesized metallic ferrofluids arise from nanometer sized suspended elemental Gd. 

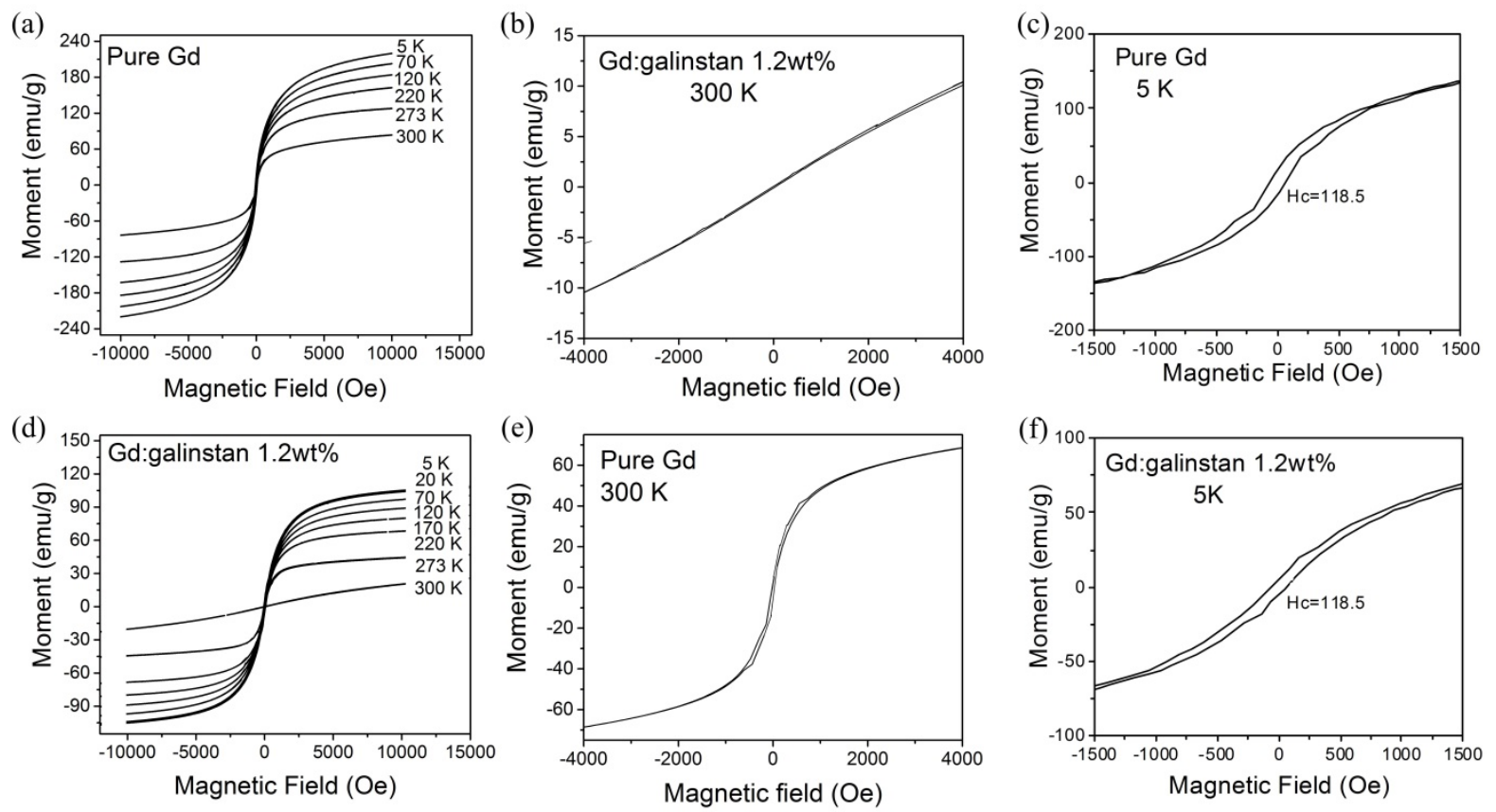

Figure 4. Magnetic hysteresis loops (MHLs) for temperatures ranging from 300 to $5 \mathrm{~K}$ under the magnetic fields of up to $2 \mathrm{~T}$ for (a) pure Gd powder, with the magnified curves for $300 \mathrm{~K}$ in (b) and $5 \mathrm{~K}$ in (c). (d) MHLs of $1.2 \mathrm{wt} \% \mathrm{Gd}$ :galinstan with the magnified curves for $300 \mathrm{~K}$ in (e) and $5 \mathrm{~K}$ in (f).

Figure 5a shows zero-field-cooled (ZFC) and field-cooled (FC) magnetization (M-T) curves of pure galinstan, $1.2 \mathrm{wt} \% \mathrm{Gd}, 2.3 \mathrm{wt} \% \mathrm{Gd}$, and pure $\mathrm{Gd}$, for temperatures ranging from $2 \mathrm{~K}$ to $300 \mathrm{~K}$ under a magnetic field of $1 \mathrm{mT}$. The magnetic signal of pure galinstan is negligible except for a superconducting transition at low temperatures $(\sim 6 \mathrm{~K}) .^{29}$ Due to the absence of a contribution of galinstan to the M-T curves, the data for the Gd:galinstan ferrofluids was normalized to the Gd content to facilitate direct comparison. The trends of the magnetization for both ferrofluids and the elemental Gd powder are consistent with each other, indicating classic ferromagnetic behavior. It is noteworthy that the magnitudes of the normalized magnetic 
moments of the metallic ferrofluids are slightly below that of pure Gd. We attribute this phenomenon to the fraction of gadolinium that is truly dissolved within the liquid melt as well as to size effects that lead to the reduction of ferromagnetic ordering within particles that feature diameters smaller than $\sim 7 \mathrm{~nm} .^{30}$

Detailed examination of the M-T curves reveals the presence of localized minimum ("dip") located at approximately $260 \mathrm{~K}$ for the Gd:galinstan ferrofluids. The position of this minimum was found to depend on the Gd concentration, occurring at lower temperatures with increased Gd concentration. This behavior and the location of the minimum was found to be in close agreement with the DSC measurements (Figure 2), leading to the conclusion that the "dip" in the M-T curves corresponds to the solidification of the liquid metal matrix.

The magnetic entropy change, $-\Delta S(T, H)$, has been determined from the magnetization curves measured at discrete temperatures and magnetic fields in the region around the ferromagnetic transition, by applying the standard Maxwell relation: ${ }^{31}$

$$
\Delta S(T, H)=S(T, H)-S(T, 0)=\int_{0}^{H} \frac{d M}{d T} d H
$$

The magnetic entropy change $\Delta S(T, H)$ could also be approximated from the isothermal magnetization curves using the numeric relation shown in equation (2): ${ }^{15,31}$

$$
-\Delta S_{M}=\sum_{i} \frac{1}{T_{i+1}-T_{i}}\left(M_{i}-M_{i+1}\right) \Delta H_{i}
$$

where $M_{i}$ and $M_{i+1}$ are the magnetic moments measured at the temperatures $T_{i}$ and $T_{i+1}$ under the magnetic field $H$. 

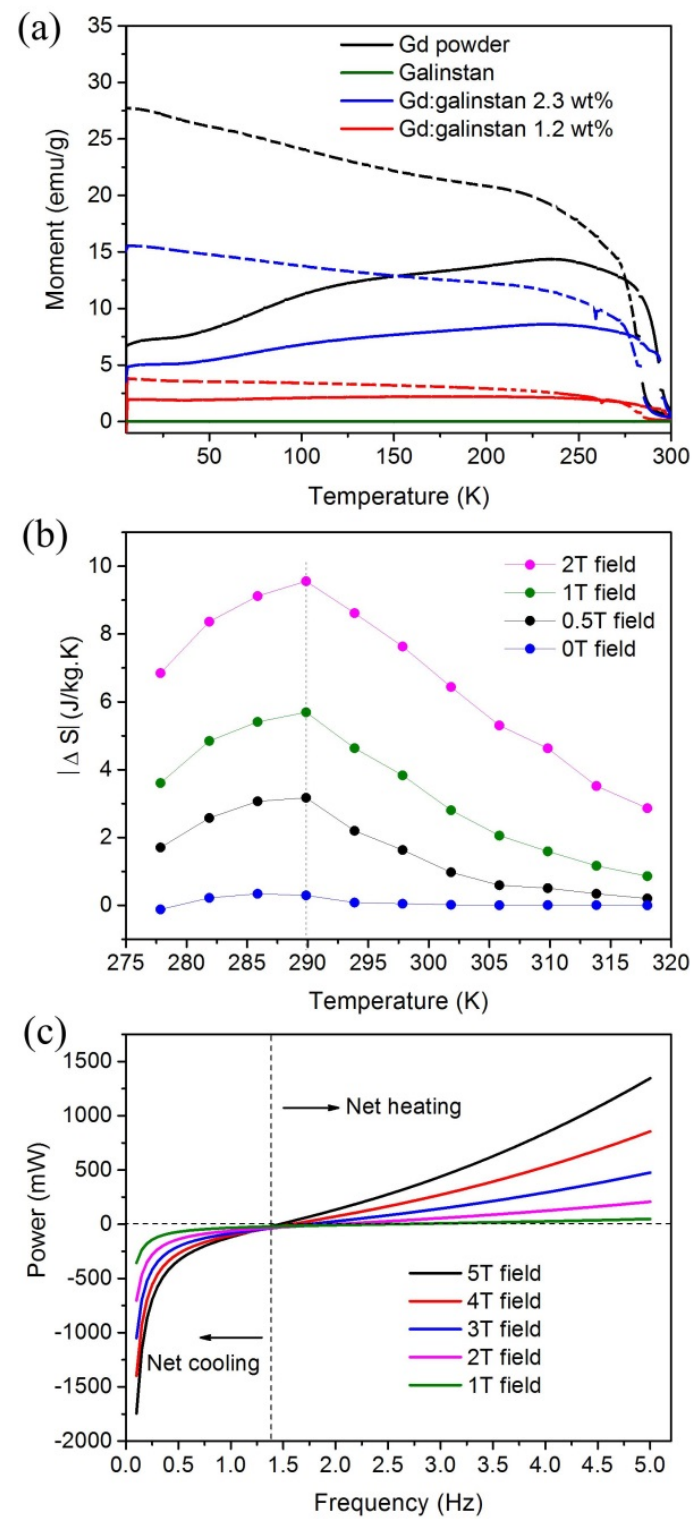

Figure 5. The zero-field-cooled (ZFC) and field-cooled (FC) magnetization (M-T) curves are represented by the continuous and dotted lines respectively in (a) for the metallic ferrofluids and pure Gd measured at temperatures ranging from 2 to $300 \mathrm{~K}$ under a magnetic field of $1 \mathrm{mT}$. (b) Calculated magnetic entropy of the $1.2 \mathrm{wt} \% \mathrm{Gd}$ :galinstan. (c) Simulated net-heat balance of a fluidic MCE device, calculated considering the determined magnetic entropy change and 
inductively induced eddy currents, for various magnetic field strengths and physical device dimensions provided in the SI. Dotted lines in (b) and (c) were added to ease readability.

Figure 5b shows the magnetic entropy change versus temperature for the metallic ferrofluid containing $1.2 \mathrm{wt} \% \mathrm{Gd}$ under magnetic fields between 0 and $2 \mathrm{~T}$, calculated utilizing Equation (2). The peak entropy change $\left|\Delta S_{M}\right|$ was found to be approximately $9 \mathrm{~J} \mathrm{~kg}^{-1} \mathrm{~K}^{-1}$ for a field strength of $2 \mathrm{~T}$ (normalized to the weight fraction of $\mathrm{Gd}$ ). This entropy change is of comparable magnitude to the magnetic entropy change observed for pure Gd powder $\left(11 \mathrm{~J} \mathrm{~kg}^{-1} \mathrm{~K}^{-1}\right){ }^{15}$ The peak position is located at $\sim 290 \mathrm{~K}$, which is close to the Curie temperature of $\mathrm{Gd}(\sim 293 \mathrm{~K}) .{ }^{15}$ The observed magnetic entropy change indicates an exploitable magnetocaloric effect in the developed liquid metal ferrofluids. Considering that the metallic matrix possesses a high thermal conductivity ( $16.5 \mathrm{~W} / \mathrm{mK}$ for pure galinstan), the liquid metal has the potential to be applied as a magnetocaloric energy conversion device at and around room temperature. ${ }^{32}$ The thermal conductivity is nearly 30 times higher than that of conventional fluids such as water $(0.61$ $\mathrm{W} / \mathrm{mK}$ ), leading to expected improved heat transfer within a potential magnetocaloric device and ultimately improved efficiency. ${ }^{9,15,32}$

To assess the potential effectiveness of the liquid metal based magnetocaloric ferrofluids, simulations were conducted to determine whether the magnetocaloric effect would overcome the competitive process associated with inductive heating that is expected to occur due to the high electric conductivity of the liquid metal (Figure 5c, see SI for details). We simulated scenarios where the liquid metal travels through a stationary magnetic field of $2 \mathrm{~T}$ at a frequency of 0.1 to $5 \mathrm{~Hz}$, which equates to linear speeds of 0.3 to $14 \mathrm{~m} / \mathrm{s}$. Magnetocaloric cooling was found to be possible at magnetization frequencies below $\sim 1.35 \mathrm{~Hz}$. Considering that efficient heat transfer 
during magnetization and subsequent demagnetization needs to be warranted in a future practical implementation of a magnetocaloric cooling device based on the herein developed materials, the proposed magnetization frequencies of approximately $1 \mathrm{~Hz}$ and below appear practically reasonable and overall encouraging.

A series of new gallium based room temperature liquid metal ferrofluids with macroscopic magnetic properties have been prepared. This was achieved following a simple manual grinding method using galinstan as a liquid metal matrix, which is utilized to dissolve elemental gadolinium. The excess gadolinium was found to precipitate within the liquid melt, forming $\sim 13$ nm suspended nanoparticles.

The developed Gd:galinstan ferrofluid containing approximately $1.2 \mathrm{wt} \%$ of $\mathrm{Gd}$ presented a reduced crystallization temperature $\left(-21.9^{\circ} \mathrm{C}\right)$ when compared with pure galinstan $\left(-14.2^{\circ} \mathrm{C}\right)$. Magnetic characterizations revealed that the Gd:galinstan ferrofluids displayed characteristic soft ferromagnetic properties, which is similar to pure Gd samples. Furthermore a large and completely reversible magnetocaloric effect $\left|\Delta \mathrm{S}_{\mathrm{M}}\right|=9 \mathrm{~J} \mathrm{~kg}^{-1}[\mathrm{Gd}] \mathrm{K}^{-1}$ (at $290 \mathrm{~K}$ and $2 \mathrm{~T}$ ) was observed for the metallic suspension. Theoretical calculations indicated the potential use of this novel MCE material for magnetic refrigeration and cooling applications, taking advantage of the high thermal conductivity of galinstan, its liquid state at temperatures below $0{ }^{\circ} \mathrm{C}$ and the magnetic properties of the suspended Gd nanoparticles. Further investigations may target suspending specially designed dispersible MCE materials such as $\mathrm{Gd}_{5}\left(\mathrm{Si}_{2} \mathrm{Ge}_{2}\right),{ }^{33} \mathrm{MnAs}^{34}$ or $\mathrm{Ni}_{55} \mathrm{Mn}_{20} \mathrm{Ga}_{25}{ }^{35}$ bestowing even larger magnetocaloric effects, into galinstan.

Apart from finding application in the field of magnetocaloric refrigeration, the developed synthetic approach may find wider applications within the field of magnetically actuated liquid metal microfluidic devices. ${ }^{36-39}$ Previous attempts to create liquid metal based ferrofluids required 
the development of core-shell particles ${ }^{11}$ where the magnetic core needed to be encapsulated with a protective oxide layer that facilitated their incorporation within the liquid melt.

The observation of the formation of nanometer sized metallic particles within the liquid melt is also of general relevance to the field of nanoparticle synthesis. Here the gallium based liquid metal fulfils the function of a reaction solvent. This finding demonstrates that low temperature liquid metals should be considered for the synthesis of nanomaterials alongside conventional molecular and ionic solvents. The electron rich and reducing environment within galinstan is particularly intriguing and justifies further exploration.

\section{Methods}

The eutectic alloy of gallium, indium and tin ,which is referred to as galinstan throughout this manuscript, was prepared in house in order to ascertain the exact composition and to avoid the presence of possible unknown proprietary components which are frequently added to commercial galinstan samples. The method used for preparing galinstan is detailed in the supporting information. The incorporation of gadolinium into the liquid melt was achieved by adding micrometer sized Gd powder (particle size $\sim 425 \mu \mathrm{m}, 99 \%$ purity Sigma Aldrich) into the galinstan. The mixture was then ground using a mortar and pestle for 30 minutes inside a $\mathrm{N}_{2}$ glovebox. Successful alloying was achieved when the liquid metal became homogeneous and the surface featured a smooth metallic mirror like appearance. The final compositions of the metallic mixtures were confirmed using SEM based EDXS.

Material Characterization: DSC was conducted using a Perkin-Elmer DSC8000 to investigate the thermal characteristics of the liquid metals within the temperature range of -50 to $50^{\circ} \mathrm{C}$. Scanning electron microscopy based microstructure and elemental analysis were conducted using a Philips XL30 SEM (1999) equipped with an Oxford X-MaxN 20 EDXS detector (2014). 
HRTEM characterization was conducted on a JEOL 2100F instrument equipped with a Gatan Orius SC1000 CCD Camera. HRTEM samples were prepared by separating suspended Gd particles within the liquid melt from the metallic ferrofluids using ultracentrifugation. This process was facilitated by placing a small droplet of the $1.2 \mathrm{wt} \% \mathrm{Gd}$ ferrofluid inside a centrifuge tube filled with DMF. The sample was centrifuged at 50,000 rcf for 20 minutes at $10^{\circ} \mathrm{C}$. The DMF phase was found to contain metallic particles after centrifugation, which was

then investigated using HRTEM. SAXS was performed on a Bruker Microcalix instrument,

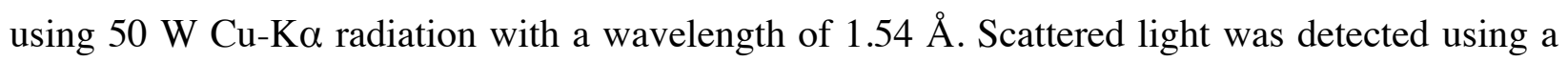
Pilatus $100 \mathrm{~K}$ detector.

Magnetic characterization of the samples was carried out in a physical property measurement system (PPMS, Quantum Design) combined with sensitive vibrating sample magnetometer (VSM). Magnetic hysteresis loops (MHLs) were measured with magnetic fields of up to 2 T.

\section{ASSOCIATED CONTENT}

Supporting information: Additional information containing experimental methods and additional experimental data are located in the supporting information. The data include Figure SI1: SEM based imaging and EDXS elemental analysis. Figure SI2: HRTEM analysis. Supplementary text detailing the modeling of a magnetocaloric device based on the developed metallic ferrofluid.

\section{AUTHOR INFORMATION}

Corresponding Authors

*E-mail: kourosh.kalantar@rmit.edu.au

*E-mail: torben.daeneke@rmit.edu.au 


\section{Author Contributions}

The manuscript was written through contributions of all authors. All authors have given approval to the final version of the manuscript.

NOTES

The authors declare no competing financial interest.

\section{ACKNOWLEDGMENTS}

The authors acknowledge the facilities, and the scientific and technical assistance of RMIT Microscopy \& Microanalysis Research Facility (RMMF) and MicroNano Research Facility (MNRF) at RMIT University. We also acknowledge the financial support from the Australian Research Council (Grant number DP170102138) and the Ministry of Science, Technology and Innovation of Brazil (CNPq) that funded Dr. Isabela Alves de Castro (Grant number 200698/2015-0).

\section{REFERENCES}

1. Smith, A.; Bahl, C. R. H.; Bjørk, R.; Engelbrecht, K.; Nielsen, K. K.; Pryds, N. Adv. Energy Mater. 2012, 2, (11), 1288-1318.

2. Nordblad, P. Nat. Mater. 2013, 12, (1), 11-12.

3. Moya, X.; Defay, E.; Heine, V.; Mathur, N. D. Nat. Phys. 2015, 11, (3), 202-205.

4. $\quad$ Moya, X.; Kar-Narayan, S.; Mathur, N. D. Nat. Mater. 2014, 13, (5), 439-450.

5. Fattori, M.; Koch, T.; Goetz, S.; Griesmaier, A.; Hensler, S.; Stuhler, J.; Pfau, T. Nat. Phys. 2006, 2, (11), 765-768.

6. Brown, G. V. J. Appl. Phys. 1976, 47, (8), 3673-3680.

7. $\quad$ Kitanovski, A.; Plaznik, U.; Tomc, U.; Poredoš, A. Int . J. Refrig . 2015, 57, 288-298.

8. Chaudhary, V.; Ramanujan, R. V. Sci.Rep. 2016, 6, 35156.

9. Lyubina, J.; Hannemann, U.; Cohen, L. F.; Ryan, M. P. Adv. Energy Mater. 2012, 2, (11), 1323-1327.

10. Zhang, Q.; Liu, J. Nano Energy 2013, 2, (5), 863-872.

11. Xiong, M.; Gao, Y.; Liu, J. J. Magn. Magn. Mater. 2014, 354, 279-283.

12. Morley, N. B.; Burris, J.; Cadwallader, L. C.; Nornberg, M. D. Rev. Sci. Instrum. 2008, $79,(5), 056107$.

13. Liu, J.; Gottschall, T.; Skokov, K. P.; Moore, J. D.; Gutfleisch, O. Nat. Mater. 2012, 11, (7), 620-626. 
14. Matsumoto, K. T.; Hiraoka, K. J. Magn. Magn. Mater. 2017, 423, 318-320.

15. Dan'kov, S. Y.; Tishin, A. M.; Pecharsky, V. K.; Gschneidner, K. A. Phys. Rev. B 1998, 57, (6), 3478-3490.

16. Palenzona, A.; Clrafici, S. Bull. Alloy Phase Diagrams 1990, 11, (1), 67-72.

17. Carey, B. J.; Ou, J. Z.; Clark, R. M.; Berean, K. J.; Zavabeti, A.; Chesman, A. S. R.; Russo, S. P.; Lau, D. W. M.; Xu, Z.-Q.; Bao, Q.; Kavehei, O.; Gibson, B. C.; Dickey, M. D.; Kaner, R. B.; Daeneke, T.; Kalantar-Zadeh, K. Nat. Commun. 2017, 8, 14482.

18. Zavabeti, A.; Ou, J. Z.; Carey, B. J.; Syed, N.; Orrell-Trigg, R.; Mayes, E. L.; Xu, C.; Kavehei, O.; O'Mullane, A. P.; Kaner, R. B.; Kalantar-zadeh, K.; Daeneke, T. Science 2017, Just accepted

19. Lyubina, J.; Schäfer, R.; Martin, N.; Schultz, L.; Gutfleisch, O. Adv. Mater. 2010, 22, (33), 3735-3739.

20. Kim, D.; Thissen, P.; Viner, G.; Lee, D.-W.; Choi, W.; Chabal, Y. J.; Lee, J.-B. ACS Appl. Mater. Interfaces 2013, 5, (1), 179-185.

21. Hemmer, P. C.; Imbro, D. Phys. Rev. A 1977, 16, (1), 380-386.

22. Jin, M.; Li, Q.; Ying, R.; Lu, X.; Jin, X.; Ding, X., Internal Friction Of Phase Transformations Observed Around Room Temperature In Ga-In-Sn Eutectic Alloys. In Arch. Metall. Mater., 2015; Vol. 60, p 2097.

23. Boldon, L.; Laliberte, F.; Liu, L. Nano Rev. 2015, 6, 25661.

24. David R. Lide, e., CRC Handbook of Chemistry and Physics, Internet Version 85 ed.; CRC Press Boca Raton, Florida, 2005.

25. Ballem, M. A.; Söderlind, F.; Nordblad, P.; Käll, P.-O.; Odén, M. Microporous Mesoporous Mater. 2013, 168, 221-224.

26. Neelima, B.; Rama Rao, N. V.; Chary, V. R.; Pandian, S. J. Alloys Compd. 2016, 661, 72-76.

27. Neikov, O. D., Chapter 2 - Mechanical Crushing and Grinding. In Handbook of NonFerrous Metal Powders, Elsevier: Oxford, 2009; pp 47-62.

28. Anderson, R.; Buscall, R.; Eldridge, R.; Mulvaney, P.; Scales, P. RSC Adv . 2014, 4, (60), 31914-31925.

29. Ren, L.; Zhuang, J.; Casillas, G.; Feng, H.; Liu, Y.; Xu, X.; Liu, Y.; Chen, J.; Du, Y.; Jiang, L.; Dou, S. X. Adv. Funct. Mater. 2016, 26, (44), 8111-8118.

30. O'Shea, M. J.; Perera, P. J. Appl. Phys. 1999, 85, (8), 4322-4324.

31. Feng-xia, H.; Bao-gen, S.; Ji-rong, S.; Zhao-hua, C.; Xi-xiang, Z. J. Phys. Condens. Matter 2000, 12, (46), L691.

32. Zhu, J. Y.; Tang, S.-Y.; Khoshmanesh, K.; Ghorbani, K. ACS Appl. Mater. Interfaces 2016, 8, (3), 2173-2180.

33. Pecharsky, V. K.; Gschneidner, J. K. A. Phys. Rev. Lett. 1997, 78, (23), 4494-4497.

34. Wada, H.; Tanabe, Y. Appl. Phys. Lett. 2001, 79, (20), 3302-3304.

35. Pasquale, M.; Sasso, C. P.; Lewis, L. H.; Giudici, L.; Lograsso, T.; Schlagel, D. Phys. Rev. B 2005, 72, (9), 094435.

36. Zhang, B.; Dong, Q.; Korman, C. E.; Li, Z.; Zaghloul, M. E. Sci. Rep. 2013, 3, 1098.

37. Li, G.; Parmar, M.; Lee, D.-W. Lab Chip 2015, 15, (3), 766-775.

38. Khoshmanesh, K.; Tang, S.-Y.; Zhu, J. Y.; Schaefer, S.; Mitchell, A.; Kalantar-zadeh, K.; Dickey, M. D. Lab Chip 2017, 17, (6), 974-993.

39. Clark, N. A. Nature 2013, 504, (7479), 229-230. 


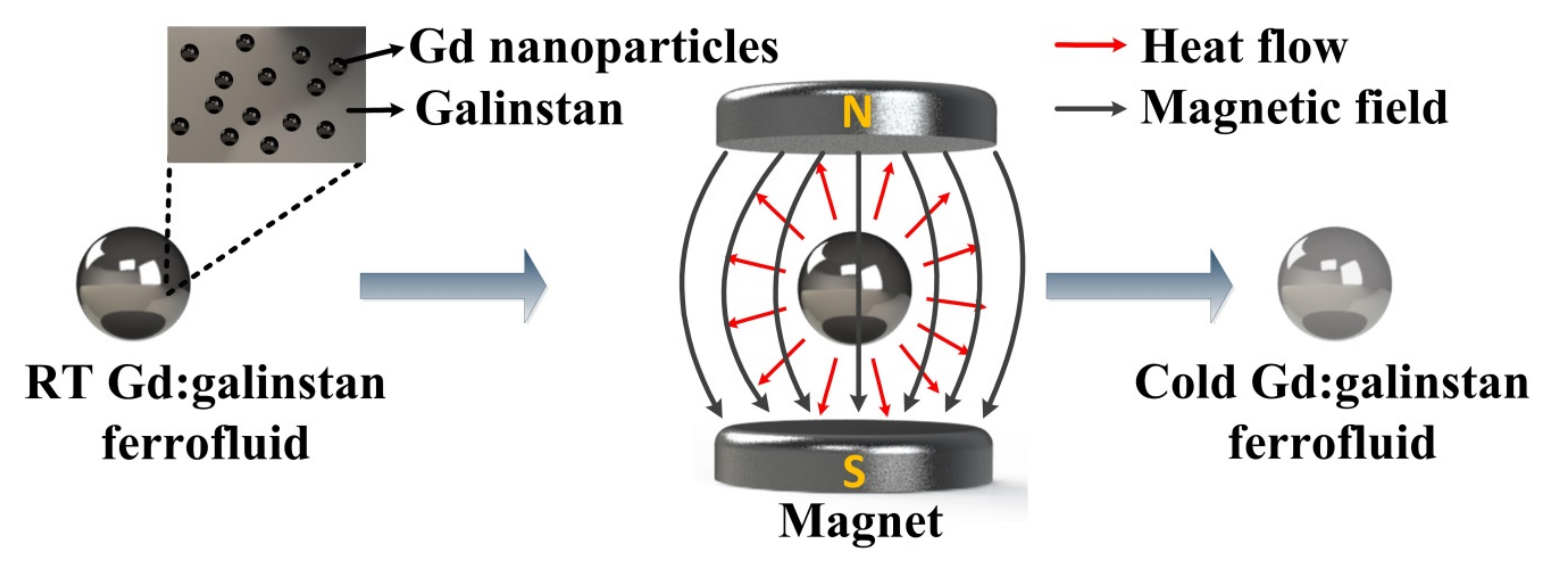


Supplementary Material 


\section{A Gallium-based Magnetocaloric Liquid Metal}

\section{Ferrofluid}

Isabela A. de Castro, ${ }^{1}$ Adam F. Chrimes, ${ }^{1}$ Ali Zavabeti, ${ }^{1}$ Kyle J. Berean, ${ }^{1}$ Benjamin J. Carey, ${ }^{1}$ Jincheng Zhuang, ${ }^{2}$ Yi Du, ${ }^{2}$ Shi X. Dou, ${ }^{2}$ Kiyonori Suzuki, ${ }^{3}$ Robert A. Shanks, ${ }^{4}$ Reece Nixon-Luke, ${ }^{5}$ Gary Bryant, ${ }^{5}$ Khashayar Khoshmanesh, ${ }^{1}$ Kourosh Kalantar-zadeh, ${ }^{1 *}$ and Torben Daeneke ${ }^{l^{*}}$

1. School of Engineering, RMIT University, Melbourne, VIC 3001, Australia

2. Institute for Superconducting and Electronic Materials, University of Wollongong, Wollongong, NSW 2500, Australia

3. Department of Materials Science and Engineering, Monash University, Clayton, VIC 3168, Australia

4. School of Science, RMIT University, Melbourne, VIC 3001, Australia

5. Centre for Molecular and Nanoscale Physics, School of Science, RMIT University, Melbourne, VIC 3001, Australia 


\section{Materials and Experimental Methods}

All materials used within this manuscript were purchased from commercial suppliers and used without further purification. Gallium (99.99\%), indium (99.99\%) and tin(99.9\%) were purchased from Roto Metals. Bulk materials and large ingots were purchased in favor of purchasing powdered samples in order to minimize the amount of pre-existing surface oxides. Gadolinium (particle size $\sim 425 \mu \mathrm{m}, 99 \%$ purity) was purchased from Sigma Aldrich.

Preparation of galinstan: The eutectic alloy of gallium, indium and tin, which is referred to as galinstan throughout the manuscript, was prepared by melting $68 \mathrm{wt} \% \mathrm{Ga}, 22 \mathrm{wt} \%$ In and 10 wt\% Sn in a beaker. The mixture was heated to $300{ }^{\circ} \mathrm{C}$, which is well above the melting temperature of the highest melting component. ${ }^{21,34}$ After complete melting, the mixture was briefly stirred with a glass rod and then allowed to cool to room temperature. The metal remained liquid at room temperature. The liquid metal was then purified by decanting the metal. This was achieved by pipetting the clean liquid metal from the center of the melt into a storage vessel, leaving the dull grey oxide skin behind. 


\section{Additional Data}
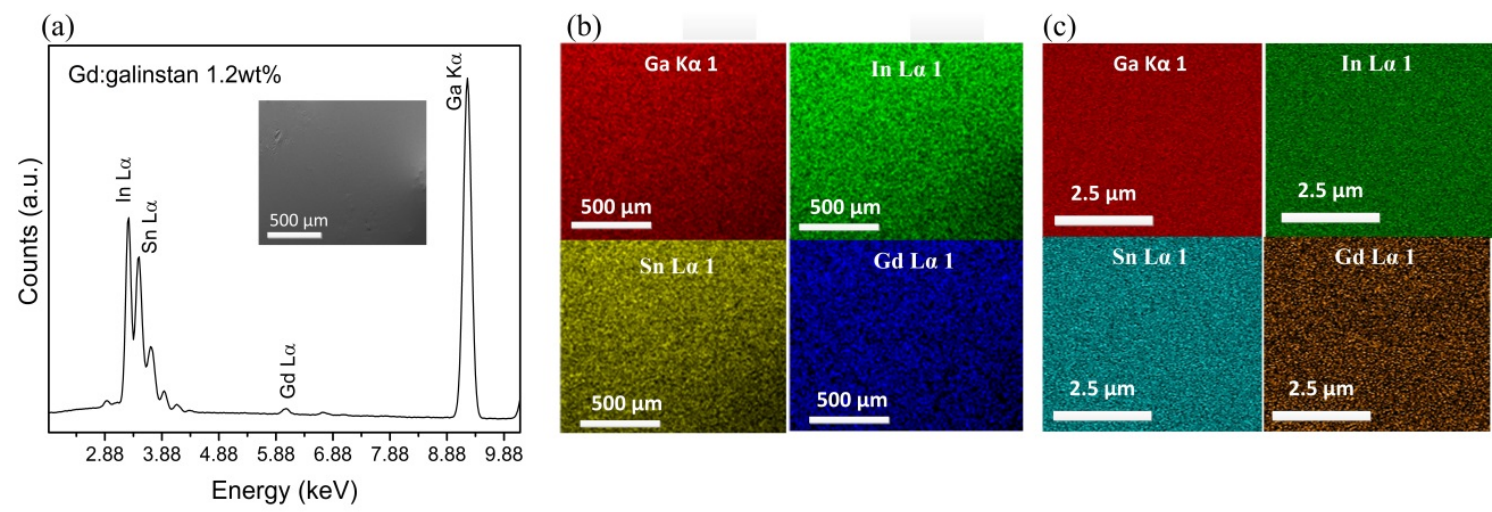

Figure SI-1: (a) Energy dispersive X-ray (EDXS) spectra, the inset features the SEM image of the investigated region of the $1.2 \mathrm{wt} \% \mathrm{Gd}$ :galinstan ferrofluid with an acceleration voltage of 30 $\mathrm{kV}$, (b-c) EDSX elemental maps corresponding to inset in SI 1 with different magnifications. 


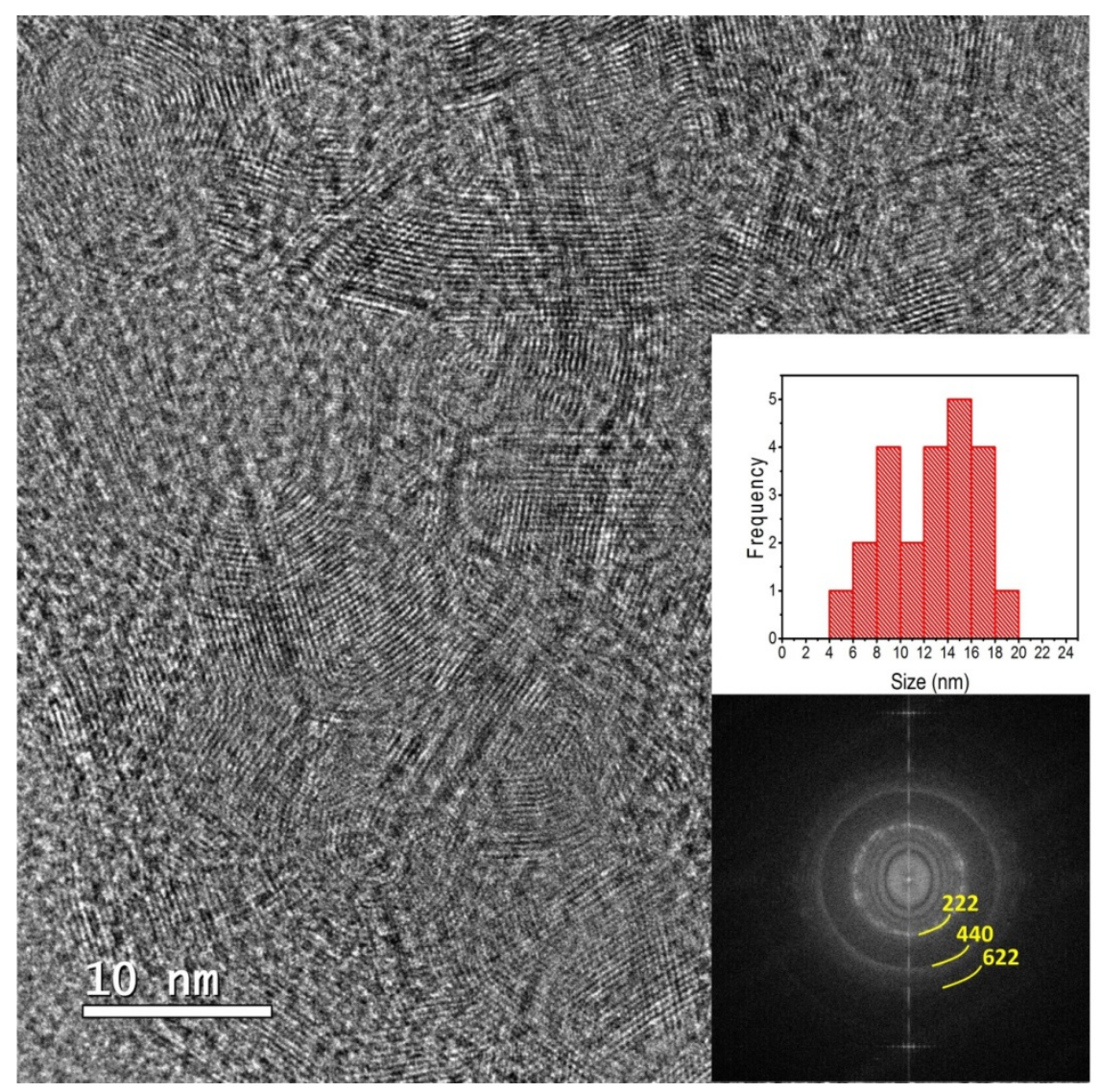

Figure SI-2: HRTEM image of particles isolated from the $1.2 \mathrm{wt} \%$ Gd:galinstan ferrofluid, showing a number of agglomerated nanoparticles featuring oxide shells with lattice fringes correspondent to cubic $\mathrm{Gd}_{2} \mathrm{O}_{3}$. The inset features particle size distribution and fast Fourier transform (FFT) of the HRTEM image with indicated lattice planes of $\mathrm{Gd}_{2} \mathrm{O}_{3}$. The particle size distribution was prepared by analysing this and additional HRTEM images (not shown). 


\section{Simulation of the internal heat budget for a magnetocaloric device based on the developed Gd:galinstan ferrofluid}

For the simulation, we assumed that the liquid metal would be applied in a magnetocaloric device with stationary magnets, while the liquid metal was pumped through the magnetic field in a closed-circuit tube system with an internal diameter of $10 \mathrm{~mm}$. For the calculation, the liquid metal was assumed to completely fill a tube with a circuit length of $100 \mathrm{~mm}$ with non-conductive media (tubing) surrounding the liquid metal. Quantitative heat transfer into an external heat exchanger was assumed during the magnetization phase (Figure 1b, step II).

The most prominent anticipated heating effect is due to the induction of eddy currents within the conductive liquid metal. The overall transferred power due to the induction of eddy currents can be calculated using the following equation: ${ }^{1}$

$$
P=\frac{\pi^{2} B_{P}^{2} d^{2} f^{2}}{6 k \rho D}
$$

where $P$ is the power per unit mass, $B_{p}$ is the peak magnetic induction, $d$ is the diameter of the liquid metal 'wire' $(0.01 \mathrm{~m}), f$ is the frequency, $k$ is a constant ( $k=2$ for a wire), $\varrho$ is the resistivity of the liquid metal $\left(2.89 \times 10^{-7} \Omega \mathrm{m}\right)^{2}$ and $D$ is the density of the material $\left(6360 \mathrm{~kg} / \mathrm{m}^{3}\right){ }^{3}$ Figure $5 c$ (main manuscript) demonstrates that the inductive energy transfer increased with increased frequency (linear speed) of the liquid metal. The heating was proportional to the strength of the magnetic field, with strong magnetic fields leading to increased heating at high pumping speeds.

The magnetic cooling due to the magnetocaloric effect has been calculated using the data determined during magnetic entropy measurements (Figure 5b, main manuscript). The overall cooling effect is obtained by integrating the magnetic entropy curve over a suitable temperature 
range which was defined as 290 to $278 \mathrm{~K}$. This temperature range resembles the expected operating temperatures for domestic refrigeration. Overall the simulation results shown in Figure $5 \mathrm{c}$ confirm that the developed gadolinium containing liquid metal can achieve overall cooling at lower pumping frequencies. Following the assumption that the competing effects of inductive heating and magnetocaloric cooling are simply additive, a net cooling effect is observed at frequencies below $1.35 \mathrm{~Hz}$. The upper frequency limit to achieve net-cooling is defined by material properties and the physical device dimensions.

\section{REFERENCES}

1. Fiorillo, F., Chapter 1 - Basic Phenomenology in Magnetic Materials. In Characterization and Measurement of Magnetic Materials, Academic Press: San Diego, 2004; pp 3-24.

2. $\quad$ Fassler, A.; Majidi, C. Adv. Mater. 2015, 27, (11), 1928-1932.

3. Morley, N. B.; Burris, J.; Cadwallader, L. C.; Nornberg, M. D. Rev. Sci. Instrum. 2008, $79,(5), 056107$. 